\title{
Association between vitamin $D$ status and cognitive impairment in acute ischemic stroke patients: a prospective cohort study
}

This article was published in the following Dove Press journal:

Clinical Interventions in Aging

\author{
Huijun Chen ${ }^{1, *}$ \\ Yuntao Liu',* \\ Guiqian Huangl,* \\ Jie Zhu ${ }^{2}$ \\ Wenqian Feng ${ }^{2}$ \\ Jincai $\mathrm{He}^{1}$
}

'Department of Neurology, The First Affiliated Hospital of Wenzhou Medical University, Wenzhou 325000, China; ${ }^{2}$ Department of Mental Health, Mental Health School, Wenzhou Medical University, Wenzhou 325000, China

*These authors contributed equally to this work
Correspondence: Jincai He

Department of Neurology, The First

Affiliated Hospital of Wenzhou Medical

University, Wenzhou 325000, Zhejiang

Province, China

Tel/fax +8657755579363

Email hjc@wmu.edu.cn
Objective: Previous studies found that low vitamin D levels were modestly associated with risk of stroke and poor functional outcome after stroke. In addition, vitamin D deficiency has been linked with cognitive decline. Our study aimed to explore the potential relationship between vitamin D levels in the short-term acute phase of ischemic stroke and cognitive impairment at 1 month.

Methods: In total, 354 ischemic stroke patients were consecutively enrolled in the study and received 1-month follow-up. The serum levels of vitamin D were measured within 24 hours after admission. Cognitive function was evaluated by the Mini-Mental State Examination (MMSE) at 1 month after acute ischemic stroke. Cognitive impairment was defined according to different education levels.

Results: According to MMSE scores, 114 participants (32.2\%) had cognitive impairment at 1 month. Patients with vitamin D deficiency were more likely to have cognitive impairment than those with vitamin D insufficiency and vitamin D sufficiency $(P<0.001)$. After adjusting for potential confounders in our Cox proportional hazards model, vitamin D deficiency was independently associated with the development of cognitive impairment in acute ischemic stroke patients.

Conclusion: Independent of established risk factors, vitamin D deficiency in the shortterm phase of ischemic stroke was associated with a higher incidence of 1-month cognitive impairment.

Keywords: vitamin D, cognitive impairment, ischemic stroke, Mini-Mental State Examination

\section{Introduction}

Cognitive impairment is a common and important complication after stroke, with prevalence ranging widely from $10 \%$ to $82 \%$. ${ }^{1}$ The presence of poststroke cognitive impairment (PSCI) has been associated with poor functional outcome, ${ }^{2}$ reduced quality of life, ${ }^{3}$ higher risk of recurrent stroke ${ }^{4}$ and aggravated economic burden. ${ }^{5}$ However, multiple factors have been found to lead to the progression of PSCI, including older age, family history, sex difference and poor educational status, ${ }^{6}$ which are not readily amenable to prevention and treatment. Therefore, it is necessary to find novel risk factors that may improve the prediction and early diagnosis of PSCI.

Vitamin D is a unique neurosteroid hormone necessary for maintaining musculoskeletal health; its deficiency is associated with many conditions including obesity, hypertension, diabetes mellitus, cardiovascular diseases (CVDs) and dementia. ${ }^{7-9}$ Accumulating evidence suggests that the vitamin D receptor (VDR) and the enzyme required for vitamin $\mathrm{D}$ activation have been found in areas of the brain essential for cognition. ${ }^{10}$ 
More recently, several experimental studies have suggested that vitamin $\mathrm{D}$ deficiency could mediate neurodegenerative processes involved in Alzheimer's disease (AD). ${ }^{11,12}$ Furthermore, previous studies found that low vitamin D levels were associated with increased odds of cognitive dysfunction in the elderly and in people with dementia. ${ }^{13-17}$ It is also reported that vitamin D deficiency is common in patients after stroke and is associated with poor functional outcome. ${ }^{18-21}$ A crosssectional study conducted in the rehabilitation period of stroke found a positive association between vitamin $\mathrm{D}$ levels and cognitive function. ${ }^{22}$ However, to date, as we know, this is the first prospective study to explore the relationship between vitamin D levels in the acute period of stroke and the development of cognitive impairment at 1 month.

In this study, we measured the serum levels of vitamin D within 24 hours after admission in patients with acute ischemic stroke and investigated the relationship of vitamin D levels with the incidence of cognitive impairment at 1 month.

\section{Materials and methods Subjects}

The study obtained the approval of the ethics committee of the First Affiliated Hospital of Wenzhou Medical University in compliance with the Declaration of Helsinki. All participants or their relatives provided written informed consents. Patients who had suffered a stroke and were admitted to the Stroke Unit of the First Affiliated Hospital of Wenzhou Medical University within 7 days were included in this study from October 2013 to February 2015. The inclusion criteria were as follows: 1) age between 18 and 80 years; 2) firstever acute stroke occurring within 7 days after stroke onset; 3) diagnosed with computerized tomography (CT) or magnetic resonance imaging (MRI) at the time of admission. The exclusion criteria were as follows: 1) primary hemorrhagic stroke or transient ischemic attack; 2) patients with any central nervous system disease such as pre-stroke dementia or severe cognitive impairment, Parkinson's disease; 3 ) severe aphasia or dysarthria, visual or auditory impairment and acute or chronic inflammatory disease; 4) patients taking vitamin D and/or calcium supplementation before stroke onset.

\section{Clinical measurements}

Demographic data included age, gender and years of education. Behavioral risk factors included body mass index (BMI), current smoking and drinking alcohol. Clinical variables included history of hypertension, diabetes mellitus, coronary artery disease (CAD), hyperlipidemia, stroke etiology as well as the serum levels of vitamin D. Stroke severity was assessed using the National Institutes of Health Stroke Scale (NIHSS) within 24 hours of admission. Functional outcomes were evaluated by the Barthel Index (BI) at discharge. CT or MRI was performed on patients within 72 hours after admission. The lesion locations of acute stroke were recorded.

\section{Psychological measurement}

Cognitive function was assessed by trained neurologists who were blinded to the subjects' clinical presentations and laboratories using the Chinese version of the Mini-Mental State Examination (MMSE) at 1 month poststroke. The MMSE has been translated into Chinese and validated for reliability and validity as a screening tool for cognitive impairment in the Chinese population. Lower scores indicate greater cognitive impairment, and cognitive impairment was defined by an MMSE score $\leq 19$ points (illiterate), $\leq 22$ points (education level of primary school), or $\leq 26$ points (education level of secondary school or above). ${ }^{23}$

\section{Laboratory tests}

Blood samples were obtained within 24 hours of hospital admission. Vitamin D status was measured by blood 25-hydroxyvitamin D [25(OH)D], the main active form of vitamin $\mathrm{D}$ and a good indicator of the whole level of vitamin D. Serum 25(OH)D levels were measured through a competitive protein binding assay at our hospital's laboratory. In both cohorts, serum $25(\mathrm{OH}) \mathrm{D}$ concentrations were divided into clinically relevant categories, vitamin $\mathrm{D}$ deficiency $(<25 \mathrm{nmol} / \mathrm{L})$, vitamin D insufficiency $(\geq 25$ to $<50 \mathrm{nmol} / \mathrm{L})$ and vitamin D sufficiency ( $\geq 50 \mathrm{nmol} / \mathrm{L}){ }^{24}$

\section{Statistical analyses}

Continuous variables were shown as mean (SD) or medians (quartiles) depending on the normal or non-normal distribution of data, while categorical variables were represented as percentages. Student's $t$-test was applied for normal distribution test, while the asymmetrically distributed variables were compared using the Mann-Whitney $U$-test, and chi-squared test was employed for proportions. In addition, Cox proportional hazards model was employed to analyze and determine the independent risk factors of PSCI, which allows adjustment for potential confounding factors. All statistical analyses were performed with SPSS for Windows, version 23.0 (SPSS Inc., Chicago, IL). A $P$-value $<0.05$ was considered statistically significant.

\section{Results \\ Baseline characteristics}

A total of 634 individuals were enrolled in the study and 410 patients were eligible for the study. Consequently, 
354 patients completed the follow-up and were included in this study (Figure 1). Among 354 patients, the mean age was $62.51 \pm 10.12$ years, and $37.3 \%$ were females; the median serum vitamin D concentration was $49.58 \mathrm{nmol} / \mathrm{mL}$ (IQR, 31.95-65.09 nmol/mL). Table 1 shows the basic characteristics of patients with and without PSCI. Among the study population, $114(32.2 \%)$ patients were diagnosed with PSCI at 1-month follow-up. Compared with non-PSCI patients, PSCI patients were more likely to be older, female and have higher NIHSS scores, lower BI scores and lower education level. Meanwhile, less cigarette smokers and alcohol drinkers were found in subjects with PSCI.

\section{Association between serum level of vitamin $\mathrm{D}$ and cognitive impairment}

Median (IQR) values of vitamin D in non-PSCI patients and PSCI patients were 51.64 (36.39-66.32) and 41.47 (19.24-57.10), respectively. Compared to those without PSCI, lower vitamin D levels were found in subjects with PSCI $(P<0.001)$. In addition, there were significant differences observed between the PSCI and non-PSCI patients according to different vitamin $\mathrm{D}$ status. Indeed, the incidence of vitamin D deficiency $(<25 \mathrm{nmol} / \mathrm{L})$ was significantly higher in the PSCI group $(P<0.001)$, whereas it was significantly lower in the PSCI group $(P=0.015)$. Moreover, Figure 2 shows a significant intergroup difference in the incidence of PSCI $(P<0.001)$. Patients with vitamin $\mathrm{D}$ deficiency were more likely to have cognitive impairment than those with vitamin $\mathrm{D}$ insufficiency and vitamin D sufficiency $(58.3 \%$ vs $28.1 \%, 26.0 \%, P<0.001)$ (Figure 2).
Table 2 shows the Cox proportional hazards model for the association between vitamin $\mathrm{D}$ status and cognitive impairment. For the whole data of stroke patients, vitamin D sufficiency was taken as a reference and the presence of PSCI taken as a dependent variable for vitamin D values; after adjusting for potential confounders, vitamin D deficiency $(<25.0 \mathrm{nmol} / \mathrm{L})$ was independently associated with the prevalence of PSCI (RR 2.124, 95\% CI 1.307-3.449, $P=0.002$ ). Moreover, years of education was significantly associated with the development of PSCI in acute ischemic stroke patients (RR 0.940, 95\% CI 0.884-0.999, $P=0.047$; Table 2).

\section{Discussion}

To the best of our knowledge, this is the first study to investigate the prospective relationship between vitamin D levels and PSCI. In our study, the main finding was that vitamin D deficiency was associated with a higher incidence of cognitive impairment in patients with acute ischemic stroke. The association remained significant after adjusting for potential confounders listed in Table 2.

In the present study, $32.2 \%$ of patients with acute ischemic stroke were diagnosed with PSCI at 1 month, which is consistent with previous findings. ${ }^{25-27}$ Additionally, some studies demonstrated that education is an important factor that affects cognitive function. ${ }^{28,29}$ Previous studies have discovered that education had the most potent effect on MMSE performance, which would influence individuals' capability of comprehension and execution, or might reflect the innate intelligence status that inhibited the subjects to complete higher levels of education. ${ }^{30}$ Our study found that education has a protective effect to cognitive function, which was consistent with previous studies. ${ }^{31}$ The addition of serum

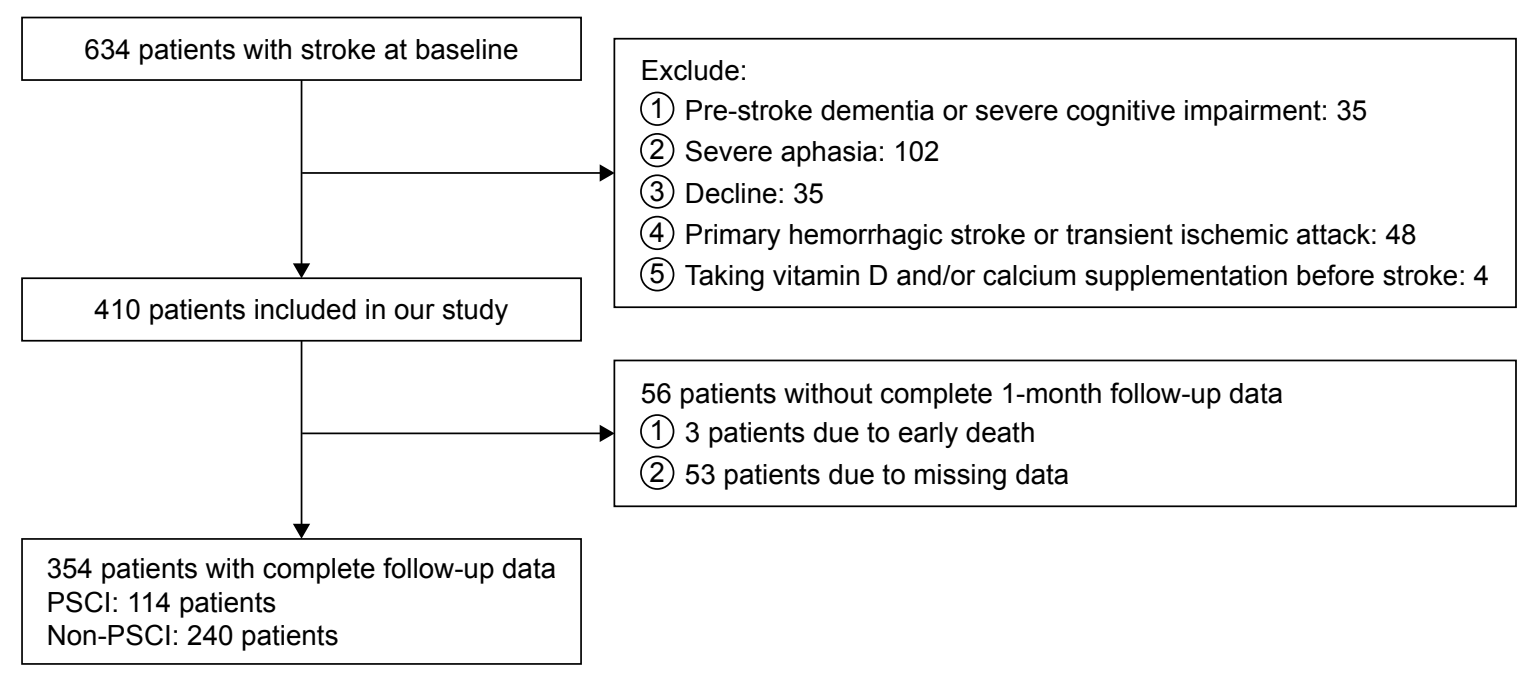

Figure I Study recruitment profile.

Abbreviation: $\mathrm{PSCl}$, poststroke cognitive impairment. 
Table I Clinical and demographic characteristics of the samples under study

\begin{tabular}{|c|c|c|c|}
\hline Baseline characteristics & Non-PSCl patients $(n=240)$ & PSCI patients $(n=1 \mid 4)$ & P-value \\
\hline Age, years, mean $\pm S D$ & $61.22 \pm 9.72$ & $65.24 \pm 10.44$ & $<0.001$ \\
\hline Female, n (\%) & $68(28.3)$ & $64(56.1)$ & $<0.001$ \\
\hline Years of education (years, IQR) & $5(2-8)$ & I (0-6) & $<0.00 \mathrm{I}$ \\
\hline $\mathrm{SBP}(\mathrm{mmHg})$ & $|56.4| \pm 20.83$ & $157.36 \pm 24.19$ & 0.720 \\
\hline $\mathrm{DBP}(\mathrm{mmHg})$ & $84.22 \pm 13.45$ & $82.61 \pm 12.40$ & 0.284 \\
\hline BMI $\left(\mathrm{kg} / \mathrm{m}^{2}\right)$, mean $\pm \mathrm{SD}$ & $24.49 \pm 3.10$ & $23.96 \pm 2.97$ & 0.134 \\
\hline Hypertension, n (\%) & 175 (72.9) & $76(66.7)$ & 0.226 \\
\hline Diabetes mellitus, n (\%) & $50(20.8)$ & $27(23.7)$ & 0.544 \\
\hline Coronary artery disease, $\mathrm{n}(\%)$ & $16(6.7)$ & $8(7.0)$ & 0.902 \\
\hline Hyperlipidemia, n (\%) & $28(11.7)$ & $9(7.9)$ & 0.278 \\
\hline \multicolumn{4}{|l|}{ Lesion location, n (\%) } \\
\hline Frontal lobe & $44(18.3)$ & $27(23.7)$ & 0.240 \\
\hline Parietal lobe & $37(15.4)$ & $18(15.8)$ & 0.928 \\
\hline Temporal lobe & $19(7.9)$ & $13(11.4)$ & 0.285 \\
\hline Occipital lobe & $25(10.4)$ & II (9.6) & 0.823 \\
\hline Basal ganglia & $106(44.2)$ & $53(46.5)$ & 0.681 \\
\hline Brainstem & $42(17.5)$ & $18(15.8)$ & 0.689 \\
\hline Cerebellum & $18(7.5)$ & $7(6.1)$ & 0.641 \\
\hline Other & $119(49.6)$ & $52(45.6)$ & 0.485 \\
\hline Stroke etiology, n (\%) & & & 0.164 \\
\hline Atherosclerosis & $204(85.0)$ & $86(75.4)$ & \\
\hline Cardio embolism & $9(3.7)$ & $7(6.1)$ & \\
\hline Small vessel occlusion & $23(9.6)$ & $19(16.7)$ & \\
\hline Other undetermined etiology & $4(1.7)$ & $2(1.8)$ & \\
\hline Current smoking, n (\%) & $81(33.8)$ & $20(17.5)$ & 0.002 \\
\hline Drinking alcohol, n (\%) & $91(37.9)$ & $28(24.6)$ & 0.013 \\
\hline NIHSS score at admission & $2(I-4)$ & $3(2-5)$ & 0.010 \\
\hline Bl score at discharge & $100(85-100)$ & $95(68.75-100)$ & 0.016 \\
\hline Vitamin D (nmol/L), median (IQR) & $51.64(36.39-66.32)$ & $41.47(19.24-57.10)$ & $<0.001$ \\
\hline Vitamin D deficiency ( $<25 \mathrm{nmol} / \mathrm{L}), \mathrm{n}(\%)$ & $25(10.4)$ & $35(30.7)$ & $<0.00$ I \\
\hline Vitamin D insufficiency (25-50 nmol/L), $\mathrm{n}(\%)$ & $87(36.3)$ & $34(29.8)$ & 0.234 \\
\hline Vitamin D sufficiency (>50 nmol/L), $\mathrm{n}(\%)$ & $128(53.3)$ & $45(39.5)$ & 0.015 \\
\hline
\end{tabular}

Abbreviations: BI, Barthel Index; BMI, body mass index; DBP, diastolic blood pressure; NIHSS, National Institutes of Health Stroke Scale; PSCl, poststroke cognitive impairment; SBP, systolic blood pressure.

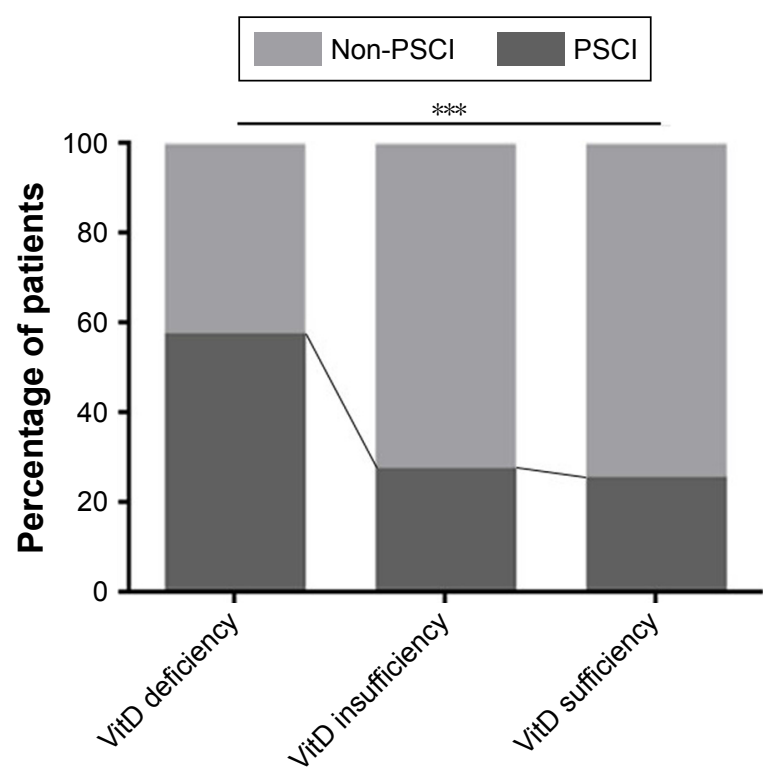

Figure 2 Percentage of patients in non-PSCl group and PSCl group according to different vitamin $D$ status.

Note: $* * * P<0.001$.

Abbreviations: $\mathrm{PSCl}$, poststroke cognitive impairment; VitD, vitamin D. vitamin D to a conventional risk factor model could increase risk prediction for PSCI.

In accordance with the present results, a growing number of studies have already reported lower vitamin D levels with cognitive impairment or dementia. ${ }^{13-17}$ As far as we know, studies investigating the possible relationship between vitamin $\mathrm{D}$ levels and the development of cognitive impairment with acute ischemic stroke were rare. In a crosssectional study conducted in Turkey, vitamin D levels were associated with cognitive impairment in stroke patients in rehabilitation period..$^{22}$ It was a study of small sample size and could not clarify the prospective relationship between vitamin D and PSCI. On that basis, we carried out the relevant study, and our research showed that vitamin D deficiency increased the risk of PSCI by 2.124-fold at 1-month follow-up, suggesting that the serum vitamin $\mathrm{D}$ might be a potential predictive marker for PSCI.

The mechanisms by which serum vitamin $\mathrm{D}$ affects cognitive function after ischemic stroke remain unclear, and 
Table 2 Cox proportional hazards model of the clinical determinants of PSCI

\begin{tabular}{|c|c|c|c|c|}
\hline & \multicolumn{2}{|l|}{ Model Ia } & \multicolumn{2}{|l|}{ Model $2^{b}$} \\
\hline & RR (95\% CI) & $P$-value & RR (95\% CI) & $P$-value \\
\hline \multicolumn{5}{|l|}{ Vitamin D levels } \\
\hline Vitamin $D$ deficiency $(<25 \mathrm{nmol} / \mathrm{L})$ & $2.243(1.442-3.448)$ & $<0.001$ & $2.124(1.307-3.449)$ & 0.002 \\
\hline Vitamin D insufficiency $(25-50 \mathrm{nmol} / \mathrm{L})$ & $1.080(0.692-1.686)$ & 0.734 & $1.096(0.687-1.747)$ & 0.701 \\
\hline Vitamin D sufficiency ( $>50 \mathrm{nmol} / \mathrm{L})$ & Reference & & Reference & \\
\hline Age, years & $1.029(1.004-1.050)$ & 0.004 & $1.018(0.997-1.040)$ & 0.099 \\
\hline Gender, female & $2.153(1.487-3.116)$ & $<0.001$ & $1.535(0.962-2.449)$ & 0.076 \\
\hline Years of education & $0.903(0.855-0.953)$ & $<0.00$ I & $0.940(0.884-0.999)$ & 0.047 \\
\hline Current smoking & $0.533(0.329-0.864)$ & 0.011 & $0.750(0.431-1.304)$ & 0.308 \\
\hline
\end{tabular}

Notes: aModel I: unadjusted. 'Model 2: adjusted for age, gender, years of education, hypertension, diabetes mellitus, coronary artery disease, current smoking, and lesion location.

Abbreviations: $\mathrm{Cl}$, confidence interval; $\mathrm{PSCl}$, poststroke cognitive impairment; $\mathrm{RR}$, risk ratio.

growing evidence suggests that vitamin D plays an important role in brain health and function. ${ }^{17}$ Vitamin D exerts various effects on brain through VDR and the vitamin D activating enzyme 1a-hydroxylase, which are broadly present in both neurons and glial cells in many areas of human brain critical for cognition. ${ }^{32,33}$ Vitamin D is involved in preventing neurodegeneration through several pathways including antioxidative mechanisms, modulation of immune response, regulation of calcium homeostasis, inhibition of pro-inflammatory agents and detoxification..$^{10,12,34,35}$ Calcitriol $(1,25-(\mathrm{OH}) 2 \mathrm{D} 3)$ is the active metabolite of vitamin D3 and attenuates the production of nitric oxide through inhibiting the expression of inducible nitric oxide synthase in the spinal cord and brain. ${ }^{36}$ High nitric oxide levels are deemed to participate in inflammatory disorders, neurodegenerative disorders and neurotoxicity. ${ }^{37}$ Besides, low vitamin D levels are known to affect macrophage and lymphocyte activity in atherosclerotic plaques and to facilitate chronic inflammation in the artery wall. ${ }^{38}$ Atherosclerosis and inflammatory reaction of artery wall play a pivotal role in the occurrence of cognitive impairment. ${ }^{27}$ It is known that vitamin $\mathrm{D}$ is inversely associated with increased cardiovascular (CV) risk itself and $\mathrm{CV}$ risk factors, which are known risk factors for cognitive impairment and dementia, including arterial hypertension, endothelial dysfunction and atherosclerosis. ${ }^{39}$ In addition, vitamin D stimulates neurogenesis and upregulates the synthesis of several neurotrophin factors important for the survival, development and functioning of neurons..$^{40,41}$ Vitamin D is considered to be involved in neurophysiology at the level of gene transcription, in regulating multiple neurotransmitters, including acetylcholine and dopamine. ${ }^{32,42}$ As a result, in the case of severe hypovitaminosis $\mathrm{D}$, a reduction of nerve conduction velocity has been observed ${ }^{43}$ Therefore, the findings mentioned above indicate that vitamin D may play a critical role in the development of PSCI.
However, our study has several limitations. First, patients with aphasia or a serious condition were excluded, as well as patients who failed to measure serum levels of vitamin $D$, which might lead to an underestimation of the actual incidence of PSCI. Second, serum vitamin D was measured only once (within first 24 hours after admission), and it is necessary to conduct a further longitudinal study assessing how vitamin D levels change over time after stroke to provide better prognostic information. Third, the variable years of education might impose some bias on the results. In future studies, we should control the years of studies interval and focus more on women when defining the inclusion criteria. Fourth, malnutrition, which could have an influence of vitamin D levels, is a risk factor for poor outcomes in patients with acute ischemic stroke. ${ }^{44}$ Our study did not collect information on nutritional status, which may influence the results. Lastly, the information of medications during hospitalization was not recorded.

\section{Conclusion}

In summary, in spite of these limitations, our study demonstrates an important relationship between serum levels of vitamin D within 24 hours after admission and the development of PSCI at 1 month after stroke. Further prospective studies and randomized clinical trials are critical in examining whether supplements of vitamin D offer a potential prevention or therapeutic target for PSCI.

\section{Availability of data and materials}

The data supporting this study are available from the corresponding author for reasonable request.

\section{Abbreviations}

PSCI, poststroke cognitive impairment; CVD, cardiovascular diseases; VDR, vitamin D receptor; AD, Alzheimer's disease; $\mathrm{CT}$, computerized tomography; MRI, magnetic resonance 
imaging; BMI, body mass index; CAD, coronary artery disease; NIHSS, National Institutes of Health Stroke Scale; BI, Barthel Index; MMSE, Mini-Mental State Examination.

\section{Acknowledgments}

We thank the study participants and their relatives and the clinical staff at all participating hospitals for their support and contribution to this project.

This study was supported by the Projects of National Science Foundation of China (No 81873799), the National Key Technology Research and Development Program of the Ministry of Science and Technology of China (grant number: 2015BAI13B01) and Wenzhou Municipal Sci-Tech Bureau Program (Y20160002). These sources had no further role in study design, data collection and analysis, decision to publish, or preparation of the article.

\section{Disclosure}

The authors report no conflicts of interest in this work.

\section{References}

1. de Haan EH, Nys GM, van Zandvoort MJ. Cognitive function following stroke and vascular cognitive impairment. Curr Opin Neurol. 2006;19(6):559-564.

2. Jokinen H, Melkas S, Ylikoski R, et al. Post-stroke cognitive impairment is common even after successful clinical recovery. Eur J Neurol. 2015;22(9):1288-1294.

3. Nys GM, van Zandvoort MJ, van der Worp HB, et al. Early cognitive impairment predicts long-term depressive symptoms and quality of life after stroke. J Neurol Sci. 2006;247(2):149-156.

4. Lee M, Saver JL, Hong KS, et al. Cognitive impairment and risk of future stroke: a systematic review and meta-analysis. CMAJ. 2014; 186(14):E536-E546.

5. Sarfo FS, Akassi J, Adamu S, Obese V, Ovbiagele B. Burden and predictors of poststroke cognitive impairment in a sample of Ghanaian stroke survivors. J Stroke Cerebrovasc Dis. 2017;26(11):2553-2562.

6. Gottesman RF, Hillis AE. Predictors and assessment of cognitive dysfunction resulting from ischaemic stroke. Lancet Neurol. 2010;9(9): 895-905.

7. Barnard K, Colón-Emeric C. Extraskeletal effects of vitamin D in older adults: cardiovascular disease, mortality, mood, and cognition. Am J Geriatr Pharmacother. 2010;8(1):4-33.

8. Dobnig H, Pilz S, Scharnagl H, et al. Independent association of low serum 25-hydroxyvitamin $\mathrm{d}$ and 1,25-dihydroxyvitamin d levels with all-cause and cardiovascular mortality. Arch Intern Med. 2008;168(12):1340-1349.

9. Resnick LM, Müller FB, Laragh JH. Calcium-regulating hormones in essential hypertension. Relation to plasma renin activity and sodium metabolism. Ann Intern Med. 1986;105(5):649-654.

10. Garcion E, Wion-Barbot N, Montero-Menei CN, Berger F, Wion D. New clues about vitamin D functions in the nervous system. Trends Endocrinol Metab. 2002;13(3):100-105.

11. Annweiler C, Bartha R, Goncalves S, et al. Vitamin D-related changes in intracranial volume in older adults: a quantitative neuroimaging study. Maturitas. 2015;80(3):312-317.

12. Gezen-Ak D, Yilmazer S, Dursun E. Why vitamin D in Alzheimer's disease? The hypothesis. J Alzheimers Dis. 2014;40(2):257-269.

13. Grant WB. Does vitamin D reduce the risk of dementia? J Alzheimers Dis. 2009;17(1):151-159.
14. Pogge E, Vitamin D. Vitamin D and Alzheimer's disease: is there a link? Consult Pharm. 2010;25(7):440-450.

15. Matchar DB, Chei CL, Yin ZX, et al. Vitamin D levels and the risk of cognitive decline in Chinese elderly people: the Chinese longitudinal healthy longevity survey. J Gerontol A Biol Sci Med Sci. 2016;71(10): 1363-1368.

16. Annweiler C, Milea D, Whitson HE, et al. Vitamin D insufficiency and cognitive impairment in Asians: a multi-ethnic population-based study and meta-analysis. J Intern Med. 2016;280(3):300-311.

17. Feart C, Helmer C, Merle B, et al. Associations of lower vitamin D concentrations with cognitive decline and long-term risk of dementia and Alzheimer's disease in older adults. Alzheimers Dement. 2017;13(11):1207-1216.

18. Xu T, Zhong C, Peng Y, et al. Serum 25-hydroxyvitamin D deficiency predicts poor outcome amongst acute ischaemic stroke patients with low high density lipoprotein cholesterol. Eur J Neurol. 2016; 23(12):1763-1768.

19. Narasimhan S, Balasubramanian P. Role of vitamin $D$ in the outcome of ischemic stroke-A randomized controlled trial. J Clin Diagn Res. 2017;11(2):Cc06-Cc10.

20. Daubail B, Jacquin A, Guilland JC, et al. Serum 25-hydroxyvitamin D predicts severity and prognosis in stroke patients. Eur J Neurol. 2013;20(1):57-61.

21. Tu WJ, Zhao SJ, Xu DJ, Chen H. Serum 25-hydroxyvitamin D predicts the short-term outcomes of Chinese patients with acute ischaemic stroke. Clin Sci. 2014;126(5):339-346.

22. Yalbuzdag SA, Sarifakioglu B, Afsar SI, et al. Is 25(OH)D associated with cognitive impairment and functional improvement in stroke? A retrospective clinical study. J Stroke Cerebrovasc Dis. 2015;24(7):1479-1486.

23. Zhang Z, Hong X, Hui LI. [The minimental state examination in the Chinese residents population aged 55 years and over in the urban and rural areas of Beijing]. Chin J Neurol. 1999;32:1-7. Chinese.

24. Ross AC, Manson JE, Abrams SA, et al. The 2011 dietary reference intakes for calcium and vitamin D: what dietetics practitioners need to know. J Am Diet Assoc. 2011;111(4):524-527.

25. Davydow DS, Levine DA, Zivin K, Katon WJ, Langa KM. The association of depression, cognitive impairment without dementia, and dementia with risk of ischemic stroke: a cohort study. Psychosom Med. 2015;77(2):200-208.

26. Levine DA, Davydow DS, Hough CL, Langa KM, Rogers MA, Iwashyna TJ. Functional disability and cognitive impairment after hospitalization for myocardial infarction and stroke. Circ Cardiovasc Qual Outcomes. 2014;7(6):863-871.

27. Wang A, Liu J, Meng X, et al. Association between oxidized low-density lipoprotein and cognitive impairment in patients with ischemic stroke. Eur J Neurol. 2018;25(1):185-191.

28. Pendlebury ST, Rothwell PM. Prevalence, incidence, and factors associated with pre-stroke and post-stroke dementia: a systematic review and meta-analysis. Lancet Neurol. 2009;8(11):1006-1018.

29. Corraini P, Henderson VW, Ording AG, Pedersen L, Horváth-Puhó E, Sørensen HT. Long-term risk of dementia among survivors of ischemic or hemorrhagic stroke. Stroke. 2017;48(1):180-186.

30. Crum RM, Anthony JC, Bassett SS, Folstein MF. Population-based norms for the mini-mental state examination by age and educational level. JAMA. 1993;269(18):2386-2391.

31. Karp A, Kåreholt I, Qiu C, Bellander T, Winblad B, Fratiglioni L. Relation of education and occupation-based socioeconomic status to incident Alzheimer's disease. Am J Epidemiol. 2004;159(2):175-183.

32. Annweiler C, Schott AM, Berrut G, et al. Vitamin D and ageing: neurological issues. Neuropsychobiology. 2010;62(3):139-150.

33. Eyles DW, Smith S, Kinobe R, Hewison M, Mcgrath JJ. Distribution of the vitamin D receptor and 1 alpha-hydroxylase in human brain. J Chem Neuroanat. 2005;29(1):21-30.

34. Brouwer-Brolsma EM, de Groot LC. Vitamin D and cognition in older adults: an update of recent findings. Curr Opin Clin Nutr Metab Care. 2015;18(1):11-16. 
35. Landel V, Annweiler C, Millet P, Morello M, Féron F. Vitamin D, Cognition and Alzheimer's disease: The therapeutic benefit is in the D-Tails. J Alzheimers Dis. 2016;53(2):419-444.

36. Garcion E, Nataf S, Berod A, Darcy F, Brachet P. 1,25-Dihydroxyvitamin D3 inhibits the expression of inducible nitric oxide synthase in rat central nervous system during experimental allergic encephalomyelitis. Brain Res Mol Brain Res. 1997;45(2):255-267.

37. Dawson VL, Dawson TM. Nitric oxide actions in neurochemistry. Neurochem Int. 1996;29(2):97-110.

38. Andress DL. Vitamin D in chronic kidney disease: a systemic role for selective vitamin D receptor activation. Kidney Int. 2006;69(1): $33-43$.

39. Grübler MR, März W, Pilz S, et al. Vitamin-D concentrations, cardiovascular risk and events - a review of epidemiological evidence. Rev Endocr Metab Disord. 2017;18(2):259-272.
40. Fernandes de Abreu DA, Eyles D, Féron F. Vitamin D, a neuroimmunomodulator: implications for neurodegenerative and autoimmune diseases. Psychoneuroendocrinology. 2009;34(Suppl 1):S265-S277.

41. Schindowski K, Belarbi K, Buée L. Neurotrophic factors in Alzheimer's disease: role of axonal transport. Genes Brain Behav. 2008;7(Suppl 1): 43-56.

42. Kalueff AV, Tuohimaa P. Neurosteroid hormone vitamin D and its utility in clinical nutrition. Curr Opin Clin Nutr Metab Care. 2007;10(1) $12-19$

43. Skaria J, Katiyar BC, Srivastava TP, Dube B. Myopathy and neuropathy associated with osteomalacia. Acta Neurol Scand. 1975;51(1): 37-58.

44. Naito H, Nezu T, Hosomi N, et al. Controlling nutritional status score for predicting 3-mo functional outcome in acute ischemic stroke. Nutrition. 2018;55-56:1-6.
Clinical Interventions in Aging

\section{Publish your work in this journal}

Clinical Interventions in Aging is an international, peer-reviewed journal focusing on evidence-based reports on the value or lack thereof of treatments intended to prevent or delay the onset of maladaptive correlates of aging in human beings. This journal is indexed on PubMed Central, MedLine,

\section{Dovepress}

CAS, Scopus and the Elsevier Bibliographic databases. The manuscript management system is completely online and includes a very quick and fair peer-review system, which is all easy to use. Visit http://www.dovepress. com/testimonials.php to read real quotes from published authors. 